Supporting Information

\title{
Vacuum-Assisted Drying Process for Screen- Printable Carbon Electrodes of Perovskite Solar Cells with Enhanced Performance Based on Cuprous Thiocyanate as a Hole Transporting Layer
}

Jing Wang ${ }^{\dagger} \sharp$, Shuiping Gong ${ }^{\dagger}$, Zongqi Chen ${ }^{\dagger}$, and Songwang Yang ${ }^{*},+\ddagger$

${ }^{\dagger}$ CAS Key Laboratory of Materials for Energy Conversion, Shanghai Institute of Ceramics, Chinese Academy of Sciences, Shanghai 201899, China

$¥$ Center of Materials Science and Optoelectronics Engineering, University of Chinese Academy of Sciences, Beijing 100049, China

\section{Corresponding Author}

*Email: swyang@mail.sic.ac.cn 


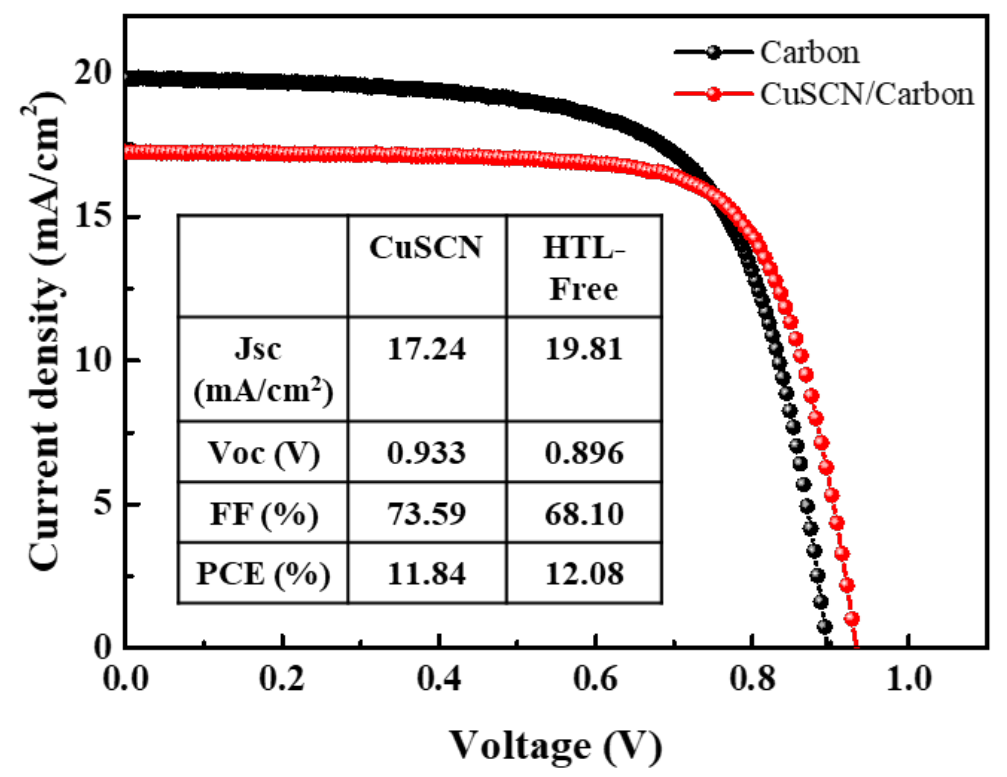

Figure S1. J-V curves of various PSCs with and without CuSCN layer.
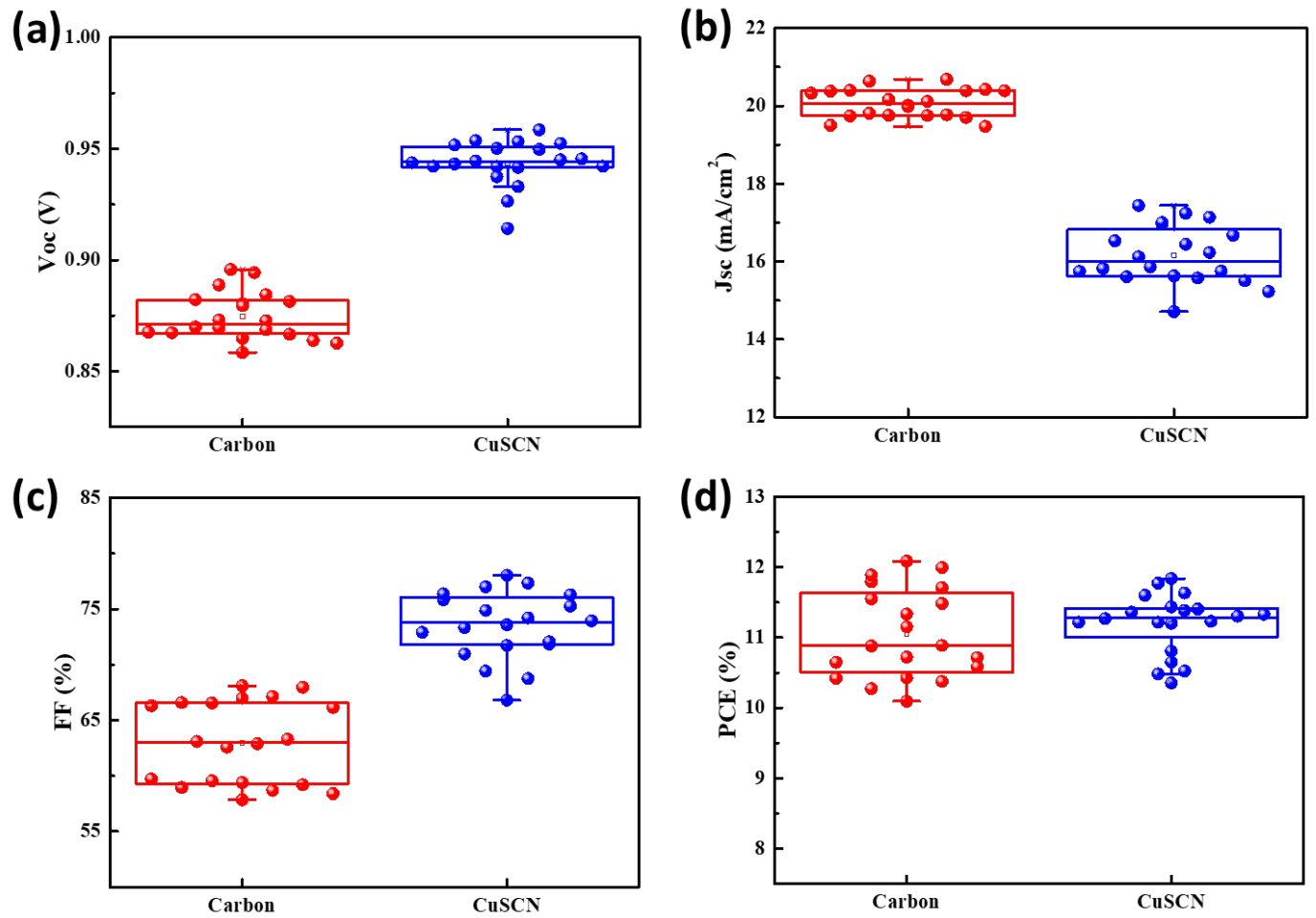

Figure S2. J-V metrics for 20 pieces of independent devices based on CuSCN layers and carbon electrodes with an active area of $0.07 \mathrm{~cm}^{2}$. 

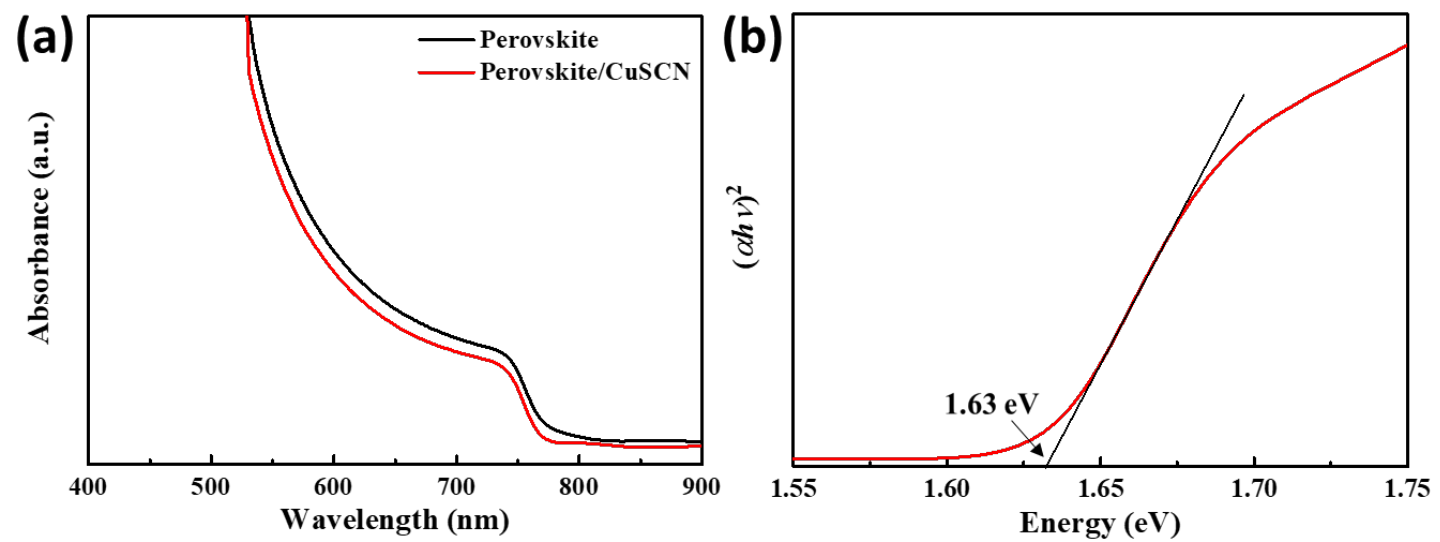

Figure S3. (a) UV-vis absorption spectra of the perovskite film and the perovskite/CuSCN film. (b) Tauc plot of the perovskite film.
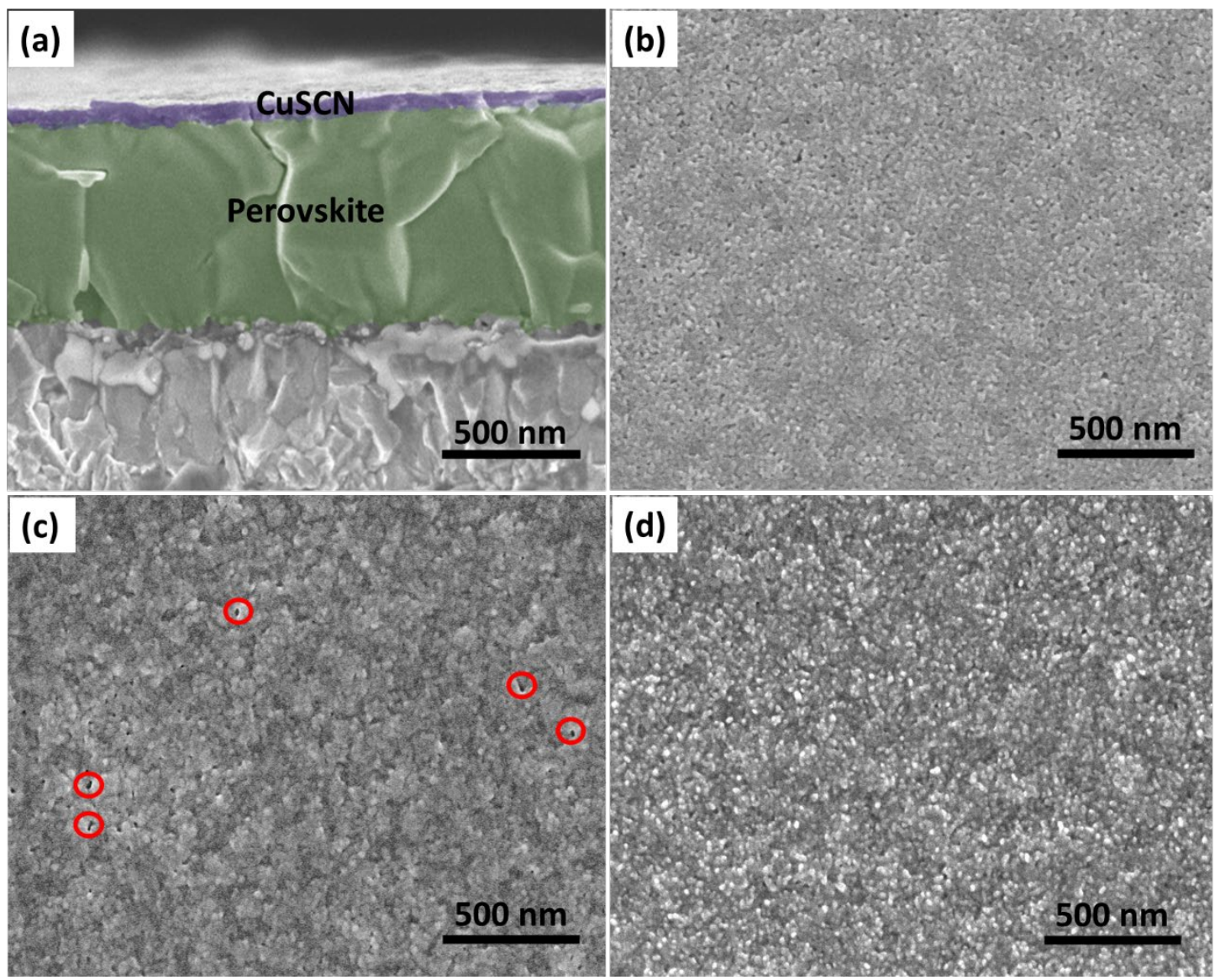

Figure S4. (a) The cross-sectional SEM image of the perovskite/CuSCN film with PDMS. The top-view SEM images of (b) the pristine CuSCN film and (c) the CuSCN film treated at $100{ }^{\circ} \mathrm{C}$ and (d) the CuSCN film treated with terpinol. 


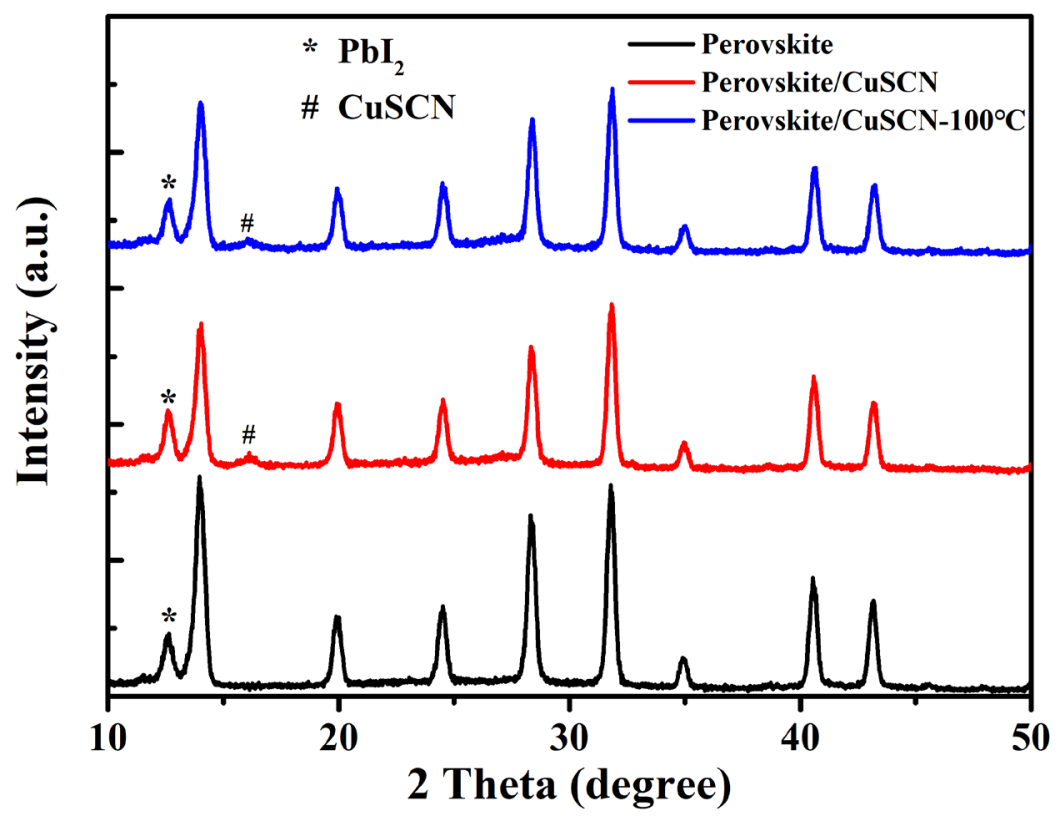

Figure S5. The grazing incident X-ray diffraction spectra of the perovskite film, the perovskite $/ \mathrm{CuSCN}$ film treated at $60^{\circ} \mathrm{C}$, and the perovskite $/ \mathrm{CuSCN}$ film treated at $100^{\circ} \mathrm{C}$.
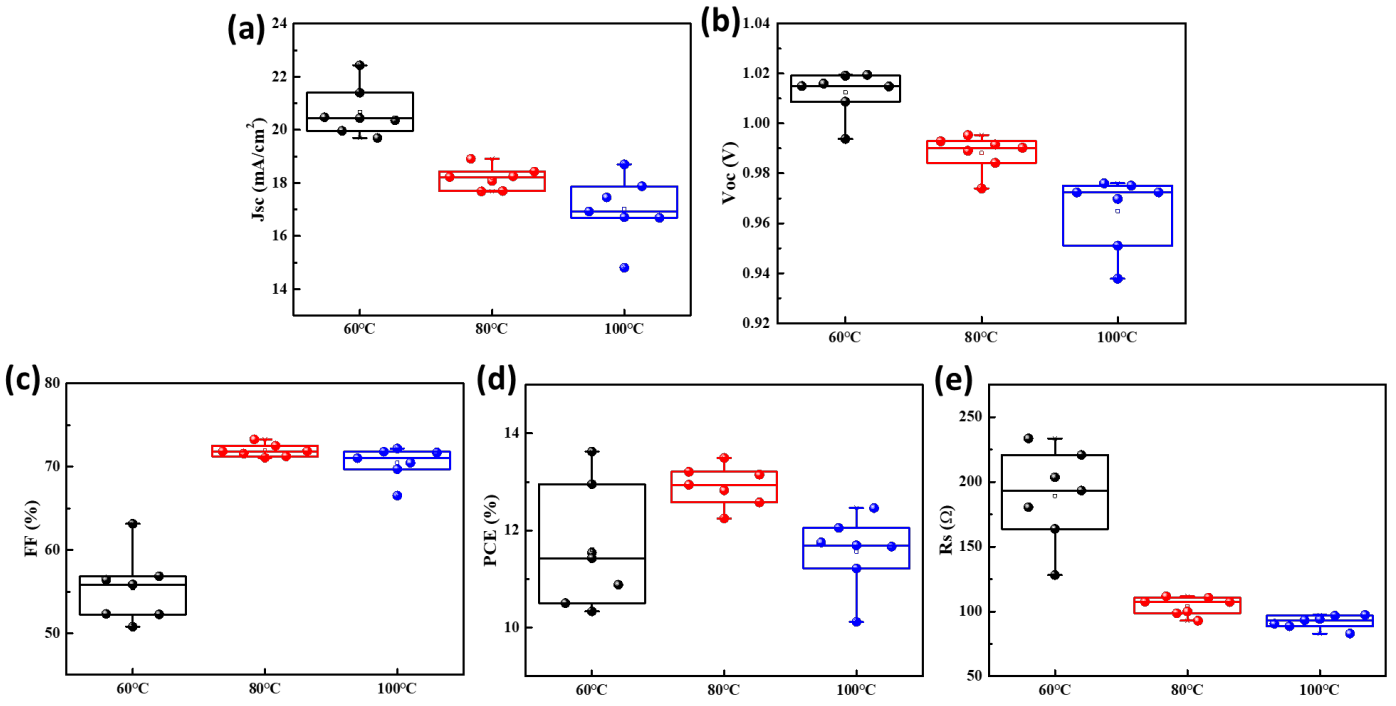

Figure S6. J-V metrics, including (a) $\mathrm{J}_{\mathrm{sc}}$, (b) $\mathrm{V}_{\mathrm{oc}}$, (c) FF, (d) PCE and (e) $\mathrm{R}_{\mathrm{s}}$ for the devices with the carbon electrode treated at different temperatures on a hot plate. 

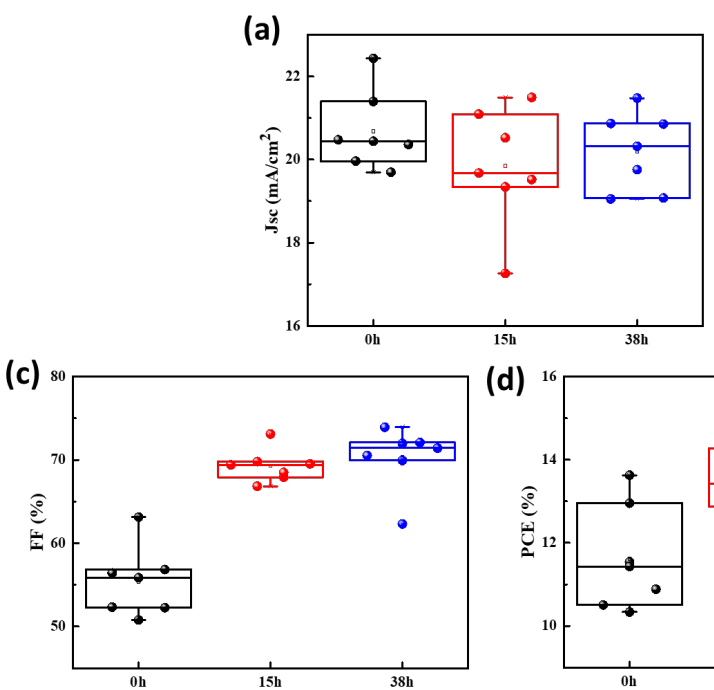
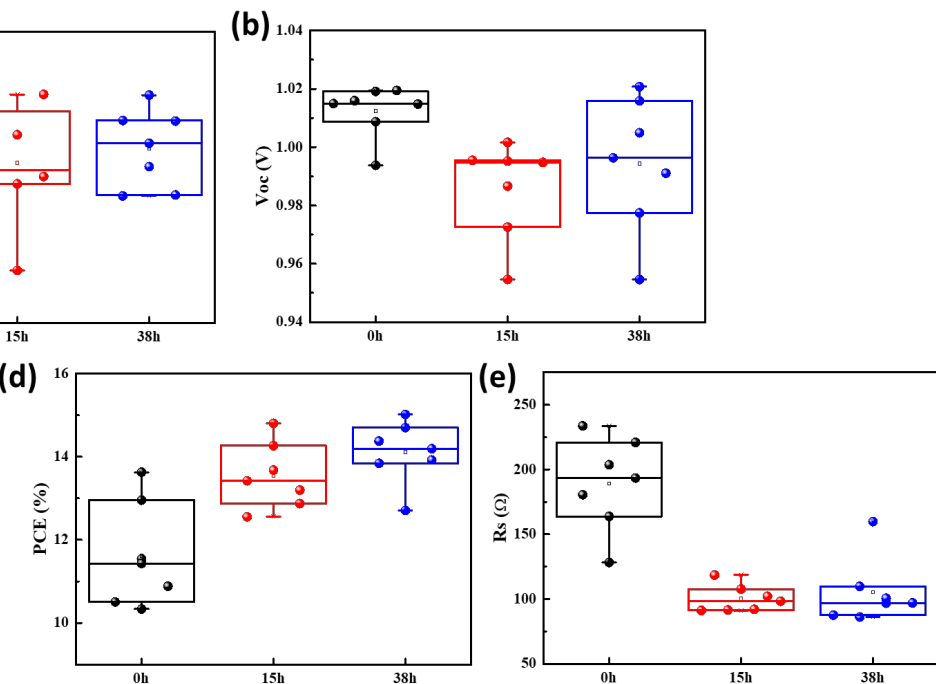

Figure S7. J-V metrics, including (a) $\mathrm{J}_{\mathrm{sc}}$, (b) $\mathrm{V}_{\mathrm{oc}}$, (c) FF, (d) PCE and (e) $\mathrm{R}_{\mathrm{s}}$ for the $60{ }^{\circ} \mathrm{C}$-treated (on a hot plate) devices with different placement time at room temperature.

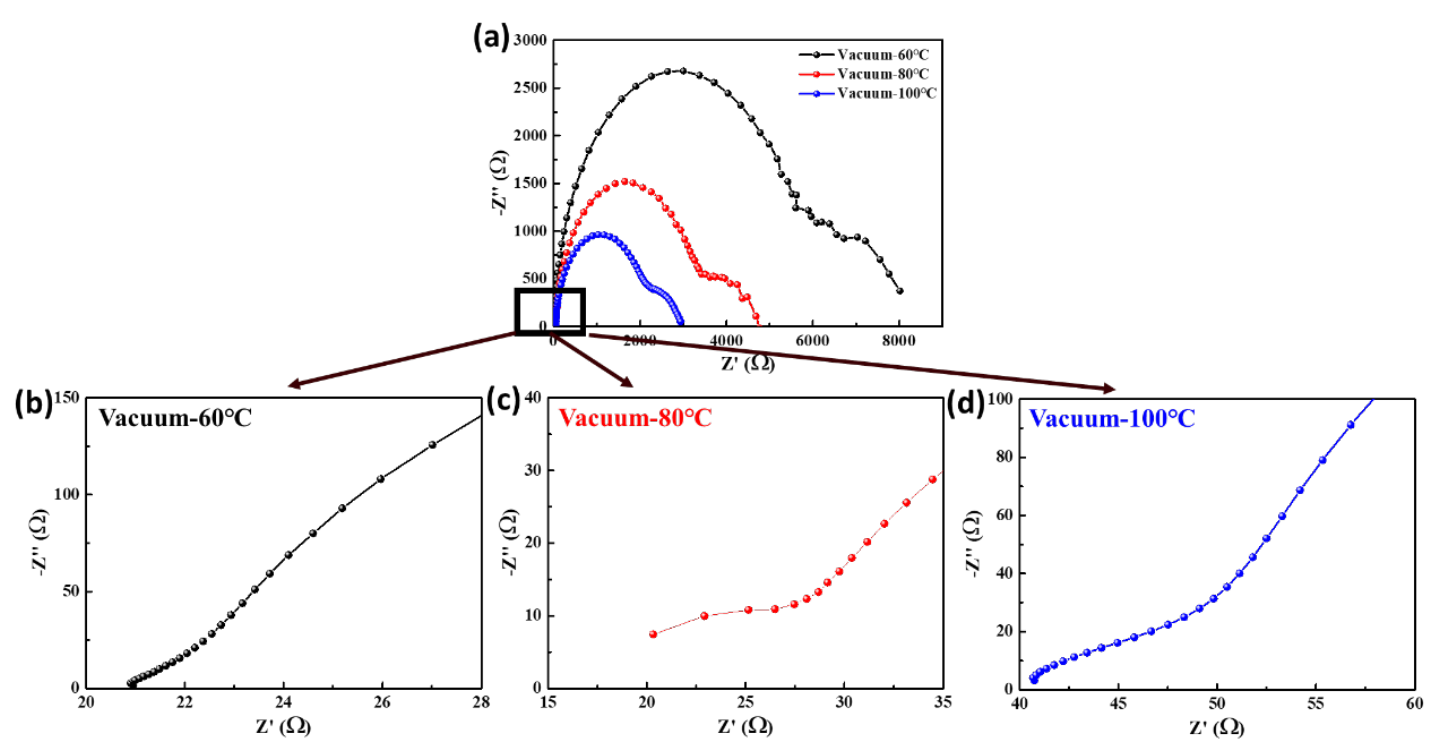

Figure S8. (a) Nyquist plots of the devices with vacuum process at different temperatures with a bias of $0.8 \mathrm{~V}$ in dark. (b), (c), and (d) Partially enlarged view of the figure (a). 

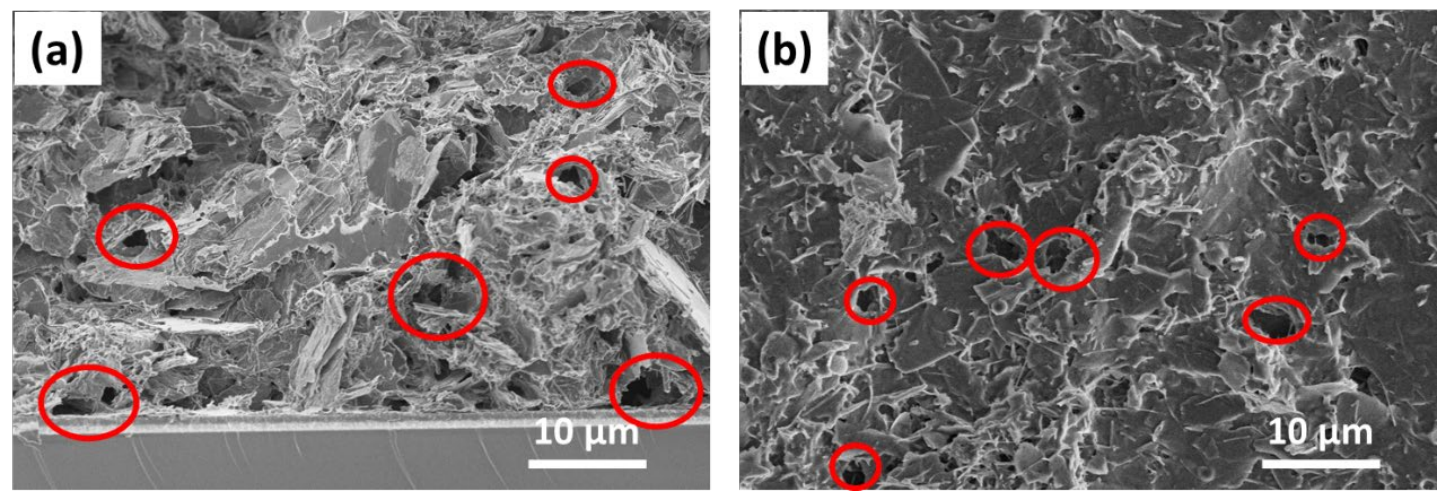

Figure S9. (a) Cross-sectional SEM image and (b) top-view SEM image of the carbon electrode 
Table S1. Summary of the parameters obtained from the fitting of the TRPL results.

\begin{tabular}{cccccc}
\hline Samples & $\mathrm{A}_{1}$ & $\tau_{1}(\mathrm{~ns})$ & $\mathrm{A}_{2}$ & $\tau_{2}(\mathrm{~ns})$ & $\tau_{\text {avg }}(\mathrm{ns})$ \\
\hline Perovskite & $\mathbf{0 . 2 3 2}$ & 6.433 & 0.619 & 287.123 & 284.78 \\
Perovskite/Carbon & 0.520 & 3.967 & 0.296 & 97.112 & 90.87 \\
Perovskite/CuSCN & 0.578 & 2.830 & 0.367 & 19.549 & 16.44 \\
\hline
\end{tabular}

Table S2. The resistance of the carbon electrodes fabricated with different methods. *

\begin{tabular}{cccccc}
\hline Printing times & 1 & 2 & 3 & 4 & 5 \\
\hline $100^{\circ} \mathrm{C}$ & $37.0 \Omega$ & $28.0 \Omega$ & $24.0 \Omega$ & $22.4 \Omega$ & $22.1 \Omega$ \\
$80{ }^{\circ} \mathrm{C}$ & $38.6 \Omega$ & $28.5 \Omega$ & $25.2 \Omega$ & $24.4 \Omega$ & $22.8 \Omega$ \\
$60^{\circ} \mathrm{C}$ & $262.2 \Omega$ & $95.5 \Omega$ & $86.2 \Omega$ & $64.4 \Omega$ & $61.8 \Omega$ \\
Vacuum-60 & & & & \\
\hline
\end{tabular}

*The resistance between the carbon electrode and the FTO electrode was simply tested by ohmmeter. 